\title{
Zero-bias molecular electronics: Exchange-correlation corrections to Landauer's formula
}

\author{
Max Koentopp and Kieron Burke \\ Department of Chemistry and Chemical Biology, Rutgers University, Piscataway, New Jersey 08854, USA \\ Ferdinand Evers \\ Institute of Nanotechnology, Forschungszentrum Karlsruhe, D-76021 Karlsruhe, Germany \\ and Institut fuer Theorie der Kondensierten Materie, Universität Karlsruhe, 76128 Karlsruhe, Germany
}

(Received 29 November 2005; published 31 March 2006)

\begin{abstract}
We show that standard first principles calculations of transport through single molecules miss exchangecorrelation corrections to the Landauer formula - the conductance is calculated at the Hartree level. Furthermore, the lack of derivative discontinuity in approximations can cause large errors for molecules weakly coupled to the electrodes. From the Kubo response theory, both the Landauer formula and these corrections in the limit of zero bias are derived and calculations are presented.
\end{abstract}

DOI: 10.1103/PhysRevB.73.121403

PACS number(s): 71.15.Mb, 31.10.+z, 73.23.-b, 73.40.-c

Much experimental progress has been made in recent years in developing methods to measure the conductance of single or few molecules in between macroscopic leads, and there is a keen interest in the theoretical modeling of such systems. ${ }^{1}$ In the case of organic molecules, covalently bound to the metallic electrodes, the transport properties are sensitive to the electronic structure, so chemical details are likely to be important, and a first principles treatment is desirable. In order to describe the coupling of the molecule to the macroscopic leads in an appropriate way, parts of the leads must be included in the calculation. Given the number of atoms required to simulate both the molecule and the first layers of the leads, density functional theory (DFT) is an obvious choice.

Since the first successful conductance experiments for single molecules, there have been several ground-breaking calculations of this type, and a variety of codes perform DFT-based calculations of $I-V$ curves of single molecules between metallic contacts. ${ }^{2-8}$ In these calculations, a potential difference $V$ between the bulk electrodes is imposed. A self-consistent ground-state Kohn-Sham (KS) calculation is performed for the molecule plus a few layers of the leads. Then, via Green's functions the current is calculated using the celebrated two-terminal Landauer formula. ${ }^{9}$ The macroscopic leads enter via self-energies. We denote this "standard" approach by its common acronym, NEGF (nonequilibrium Green's function technique). These calculations are parameter free and often yield qualitative agreement with the experiment, and so might appear to be as rigorous as any DFT calculations. But a detailed comparison for organic molecules between $\mathrm{Au}$ electrodes reveals quantitative discrepancies. Conductances are typically overestimated, often by 1 or 2 orders of magnitude. ${ }^{10}$

Neither the Hohenberg-Kohn theorem, ${ }^{11}$ which established ground-state DFT, nor the Runge-Gross theorem for time-dependent problems, ${ }^{12}$ apply to extended systems carrying current in homogeneous electric fields. In consequence, questions have recently been raised about the validity of the NEGF approach. ${ }^{10,13}$ For example, the calculated transmission is that of the KS potential. In the case of a molecule weakly coupled to two leads, whose KS levels are sharp, well-separated resonances, the NEGF approach produces peaks in the conductance at the positions of the unoccupied levels of the KS system. ${ }^{5}$ Such transitions are known to differ, in general, from the true excitations of the interacting electronic system.

To tackle the transport problem rigorously for a finite bias is daunting, and only recently have several suggestions been put forward. ${ }^{14-16}$ In the present paper, we examine only the weak bias regime, i.e., the limit in which the potential difference across the molecule is infinitesimal, because here we can deduce the exact answer.

A primary result of this paper is to rigorously demonstrate that NEGF calculations include only the Hartree response of the system. It is alarming, that this level of calculation can be inadequate already, when, e.g., the response of an isolated molecule to a static electric field is to be considered. This lack is utterly independent of which standard approximate functional is used: All DFT calculations to date suffer from this limitation. It is inherent in the methodology, just as for all Hartree calculations. Second, we estimate the size of the exchange-correlation (XC) corrections using the gradient expansion in the current of Vignale-Kohn. ${ }^{17}$ Even when such contributions are small, the lack of derivative discontinuity in semilocal functional approximations for the ground state likely produces significant errors. Finally, we argue that, under certain conditions, peak spacings in a zero-bias Coulomb blockade experiment are accurately given by NEGF calculations.

Consider any system that can carry a dc current in a specific direction (which we call the $z$ direction) and that contains some atomic-sized barriers in this direction. For simplicity, we analyze only the symmetric case. We apply a weak uniform (also for simplicity) electric field in the $z$ direction, and use time-dependent current density functional theory (TDCDFT) to calculate the current response.

The response of a system to a weak electric field is given by the Kubo formula as 


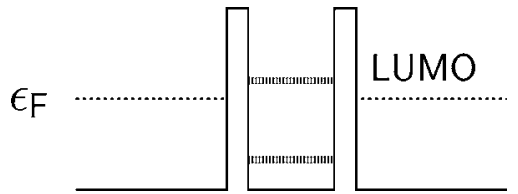

FIG. 1. Double barrier resonant tunneling cartoon of a molecule between two metallic leads; the LUMO has been shifted and broadened relative to the isolated molecule.

$$
\mathbf{j}(\mathbf{r} \omega)=\int d^{3} r^{\prime} \hat{\sigma}\left(\mathbf{r} \mathbf{r}^{\prime} \omega\right) \mathbf{E}_{\mathrm{ext}}\left(\mathbf{r}^{\prime} \omega\right)
$$

where $\mathbf{j}(\mathbf{r} \omega)$ is the first-order (physical) current density response to the external electric field, and we use atomic units throughout. Here $\hat{\sigma}\left(\mathbf{r r} \mathbf{r}^{\prime} \omega\right)$ is the frequency-dependent nonlocal conductance describing the response to the external field, rather than the response to the total electric field.

Within TDCDFT, the KS system is defined to reproduce the time-dependent current density. Thus, Eq. (1) becomes, for the KS system,

$$
\mathbf{j}(\mathbf{r} \omega)=\int d^{3} r^{\prime} \hat{\sigma}_{S}\left[n_{0}\right]\left(\mathbf{r} \mathbf{r}^{\prime} \omega\right)\left(\mathbf{E}_{\mathrm{tot}}\left(\mathbf{r}^{\prime} \omega\right)+\mathbf{E}_{\mathrm{XC}}\left(\mathbf{r}^{\prime} \omega\right)\right),
$$

where $\mathbf{j}(\mathbf{r} \omega)$ is still the exact physical current response, but now found from the KS nonlocal conductance (a functional of the ground-state density $n_{0}$ ) applied to the KS electric field, which includes both the total electric field (external plus Hartree) and the $\mathrm{XC}$ contributions.

The external field produces a finite potential drop $V$ across the barrier. ${ }^{20}$ We restrict ourselves to one dimension, to demonstrate the principle. In the limit in which $\omega \rightarrow 0$, the nonlocal conductance becomes coordinate independent. ${ }^{18,19}$ Thus, the left-hand side becomes independent of $z$, and the integral over $z^{\prime}$ applies only to the potentials,

$$
I=\sigma_{S z z}(\omega=0)\left(V+V_{\mathrm{XC}}\right),
$$

where $V$ is the integral over the external and Hartree fields and $V_{\mathrm{XC}}$ is the induced net $\mathrm{XC}$ potential drop in the vicinity of the barrier. Equation (3) and the following interpretation are the important results of this paper. We analyze it in two steps.

(i) Ignore $V_{\mathrm{XC}}$ : in the absence of the $\mathrm{XC}$ potential drop, Eq. (3) tells us that the conductance, $I-V$, is just that of the ground-state $\mathrm{KS}$ system. Careful derivations ${ }^{18}$ show that, for noninteracting systems,

$$
\sigma_{S z z}(\omega=0)=T_{S}\left(\epsilon_{F}\right) / \pi,
$$

where $T(\epsilon)$ is the transmission through all channels through the barrier. The resonances in the KS transmission function translate into peaks in the conductance for the interacting system without correction.

This brings us to the problem mentioned in the second paragraph, namely, the positions of the resonances in the NEGF approach compared to the physical system. Imagine the case of a one-dimensional double barrier, as shown in Fig. 1 as the "molecule." Usually, $\epsilon_{F}$ is located in a spectral gap of the molecule, (as in Fig. 1), so that the system is

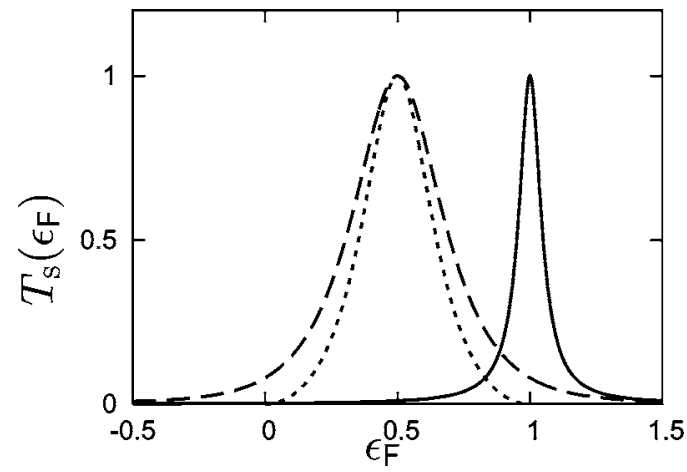

FIG. 2. Conductance peak from resonance: the dashed line is the self-consistent approximate functional result, the dotted line is the approximate result as $\gamma \rightarrow 0$, and the solid line is the exact result. Here $\epsilon_{L}=0, \Delta \epsilon=1$, and $\gamma_{0}=0.1$.

off-resonance and the conductance is nonvanishing only due to the small overlap between the very weakly broadened levels and $\epsilon_{F}$.

To probe the unoccupied resonances at zero bias, apply a gate voltage $V_{g}$ to the molecule perpendicular to the leads, shifting the lowest unoccupied molecular orbital (LUMO) down to $\epsilon_{F}$. As it passes through $\epsilon_{F}$ (as a function of $V_{g}$ ), there will be a large peak in the conductance. But consider what happens when the resonance begins to overlap with $\epsilon_{F}$. By virtue of its $n_{0}$ dependence, the exact $\mathrm{KS}$ ground-state potential differs significantly from the off-resonant case, altering the transmission characteristics. Peaks in transmission are not at the position of $\left(V_{g}=0\right)$-unoccupied resonances.

For a sharp resonance, the transmission coefficient is given by

$$
T(\epsilon)=\left(\frac{\gamma}{2}\right)^{2} /\left\{\left(\epsilon-\epsilon_{\mathrm{res}}\right)^{2}+\left(\frac{\gamma}{2}\right)^{2}\right\},
$$

where $\epsilon_{\mathrm{res}}$ and $\gamma$ are the position and width of the resonance, which, in DFT, depend on the partial occupation, $0 \leqslant f \leqslant 1$, of the resonant level. We will now see how the use of smooth, approximate density functionals influences the position and width of the resonance.

Using the spectral function $A(E)$ we can write expressions for the spectral density of states, $n(\epsilon)=\frac{1}{\pi} A$, as well as for the transmission $T=\frac{\gamma}{2} A$, to obtain a simple linear relationship between $n(\epsilon)$ and the transmission of such a level, $n(\epsilon)$ $=2 T(\epsilon) /(\gamma \pi)$. The self-consistent $f$ is found from integrating over $n(\epsilon)$ as

$$
f=\int_{-\infty}^{\epsilon_{F}} d \epsilon n(\epsilon)=\frac{1}{2}+\frac{1}{\pi} \tan ^{-1}\left\{2 \frac{\epsilon_{F}-\epsilon_{\mathrm{res}}(f)}{\gamma(f)}\right\} .
$$

After inverting this,

$$
T^{-1}\left(\epsilon_{F}\right)=1+\tan ^{2}\left\{\pi\left(f\left(\epsilon_{F}\right)-1 / 2\right)\right\} .
$$

In Fig. 2, we plot the transmission over energy for this situation, with the parameters given in the caption. For this calculation we set the width constant $\left(\gamma(f)=\gamma_{0}\right)$. The actual dependence of $\gamma$ on $f$ is expected to be weak and have little effect on transmission peaks. Now $\Delta \epsilon=\epsilon_{H}-\epsilon_{L}$ is several $\mathrm{eV}$, 
where $\epsilon_{H}$ is the highest occupied orbital of the $N+1$-electron molecule, and $\epsilon_{L}$ is the LUMO of the $N$-electron molecule. As in a NEGF calculation using a semilocal functional, $\epsilon_{\text {res }}$ always depends smoothly on $f$, and varies continuously between $\epsilon_{L}$ and $\epsilon_{H}$, we obtain (assuming $\epsilon_{\mathrm{res}}=\epsilon_{L}+f \Delta \epsilon$ ) the dashed line.

For weakly coupled leads, where $\gamma \ll \Delta \epsilon$ (at any occupation), the Fermi level is pinned to the resonance $\left(\epsilon_{\mathrm{res}}(f)\right.$ $\rightarrow \epsilon_{F}$ ) for $f \neq 0$ or 1 , so $\epsilon_{F}=\epsilon_{L}+\Delta \epsilon f$ and we obtain, using Eq. (7) the dotted line in Fig. 2. Thus, in an NEGF calculation, Eq. (7) always produces a broad peak whose width is comparable to $\Delta \epsilon$. For the case of a linear relation, the width is just $\Delta \epsilon / 2$.

But this is entirely an artifact of smooth density functional approximations. The real system has a sharp resonance centered at $\epsilon_{H}$. The exact KS potential of the molecule jumps (relative to the reservoir) as soon as there is an infinitesimal occupation of the resonant level,

$$
\epsilon_{\text {res }}=\epsilon_{L}+\Theta(f+\eta) \Delta \epsilon \quad(\eta \rightarrow 0) .
$$

Solving Eq. (6) for $\epsilon_{F}$, we obtain a peak in the transmission of width $\gamma$ around $\epsilon_{H}$-the solid line in Fig. 2. This is the famous derivative discontinuity ${ }^{21}$ of DFT.

Since the true transmission will be much more narrowly peaked than that in the approximate DFT calculation, if the system is off-resonance, the DFT calculation produces a strong overestimate of the true conductance. Figure 2 is a cartoon of this situation, in which the width of the resonance is $10 \%$ of the level shift, and a severe overestimate occurs if the Fermi level is at, e.g., 0.5.

In reality, organic molecules may not be so weakly coupled to the leads (although the widths of resonances in GGA calculations are not a sure indicator of this, for the reasons given above). But this effect, or some remnant of it at less weak coupling, would explain the severe overestimate. In Ref. 10, the conductance of benzene was calculated in two ways, (a) by employing the standard approach based on the KS energies and orbitals of an equilibrium DFT calculation (GGA) and (b) by replacing the KS data with their counterparts obtained from a Hartree-Fock analysis. The typical transmission level in a window of $2 \mathrm{eV}$ about $\epsilon_{F}$ was reduced by an order of magnitude. The best current way to see if this effect is the culprit for the overestimate would be to perform exact exchange calculations ${ }^{22}$ which should contain most of the effects of the true discontinuity.

(ii) Include $V_{\mathrm{XC}}$ : Now we discuss how to include

$$
V_{\mathrm{XC}}=\int d z \mathbf{E}_{z, \mathrm{XC}} \quad(z, \omega \rightarrow 0),
$$

where $\mathbf{E}_{\mathrm{XC}}$ is the XC electric field induced in response to the applied field (and ignoring the coupling between the longitudinal and transverse modes in $\sigma_{S}$ ). We first note that, for any pure density functional, $\mathbf{E}_{\mathrm{XC}}=-\nabla \cdot \delta v_{\mathrm{XC}}$, so that $V_{\mathrm{XC}}$ $=\delta v_{\mathrm{XC}}(z \rightarrow \infty)-\delta v_{\mathrm{XC}}(z \rightarrow-\infty)$, i.e., the net induced XC potential drop from the extreme left to the extreme right of the barrier. In any semilocal approximation, $V_{\mathrm{XC}}$ therefore vanishes identically, as the induced density response is localized to the region of the barrier, so that far from the barrier,

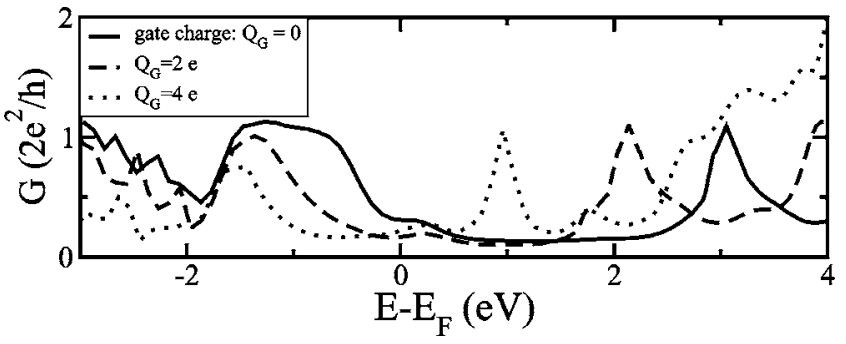

FIG. 3. Transmission of benzene-dithiolate in the presence of a gate. The gate is realized by a square sheet of homogenously distributed dummy charges (total sum is denoted by $Q_{G}$ ) located a distance of $3.8 \AA$ above the carbon ring.

$\delta v_{\mathrm{XC}}=0$. Thus, using common density functionals, the corrections to the KS Landauer formula vanish.

The corrections are produced by nonlocal interactions present in the exact XC functional. At least two mechanisms are well established, which generate such interaction terms that are nonlocal in the density. The most obvious one is exact exchange. Even a simple static exchange calculation, ${ }^{22}$ including response terms (i.e., beyond NEGF), might yield a finite result, i.e., a net drop in the exchange potential across the barrier, just as Hartree does. A second mechanism is of the hydrodynamical type and therefore finds its natural description within TDCDFT. For this case we are able to offer an analytical estimate for the size of the effect. In the spirit of Ref. 23, we use the Vignale-Kohn (VK) approximation ${ }^{17}$ to obtain an expression for the purely viscous contribution to $V_{\mathrm{XC}}$ (although the original derivation assumes a highfrequency regime). It involves a spatial variation of the density, $n(\mathbf{x})$, which originates from the backscattering off the barrier. A rough estimate is obtained by assuming (a) that the most important variations in the density profile along the wire are of the Friedel type and (b) that the viscosity can be approximated by its static, homogenous value characteristic of a three-dimensional Fermi liquid. With these simplifications Eq. (9) can be rewritten as

$$
V_{\mathrm{XC}} / V \approx-\left(1-T\left(\epsilon_{F}\right)\right) T\left(\epsilon_{F}\right) /\left(40 \pi^{1 / 2} k_{F}^{3 / 2}\right) .
$$

The viscosity counteracts the current flow and reduces the conductance. The factor $(1-T)$ takes into account that a barrier causing reflection (and thus density inhomogeneities) is needed for viscous flow to be generated.

Because $k_{F} \approx 1$, the small prefactor (together with the fact that $T \leqslant 1$ for well-resolved resonances) guarantees only small corrections, as was found in a recent calculation. ${ }^{24}$ This result, though suggestive, is not rigorous, however, because it ignores both the elastic hydrodynamic contribution and the limited validity of VK.

Finally, we show how, despite the fact that XC corrections to the voltage $\left[V_{\mathrm{XC}}\right.$ in Eq. (3)] are missing, NEGF might be used to obtain exact information to be compared with experiment. Consider Coulomb blockade experiments, which measure zero-bias conductance as a function of $V_{g}{ }^{25}$ In Fig. 3 we show the transmission of a benzene ring, coupled via two sulphur atoms to gold electrodes, obtained from a NEGF transport calculation. ${ }^{26}$ The KS LUMO moves toward $\epsilon_{F}$ 
with increasing gate charge (voltage). As argued above, the position of the LUMO does not give a reliable estimate for the real peak position. However, at the particular $V_{g}$ where the LUMO passes through $\epsilon_{F}$, its energy must coincide with the real many-body level. (In Fig. 3, this would correspond to $Q_{G} \approx 6$.) Therefore, at those $V_{g}$ where a KS level crosses $\epsilon_{F}$, a peak in the $I-V$ characteristics is observed. The peak spacings are given by a ground-state DFT calculation.

Our calculation demonstrates the principle. It is missing the derivative discontinuity, but at least we can ignore the missing $V_{\mathrm{XC}}$. There may be cases where the derivative discontinuity is unimportant (i.e., for strong coupling or larger molecules) but the missing $V_{\mathrm{XC}}$ is not. Then standard NEGF calculations will yield accurate results for peak spacings in Coulomb blockade experiments, although the peak heights are strongly overestimated.
A not unlikely scenario, in which the $V_{\mathrm{XC}}$ corrections are especially large is as follows. Suppose that the quasistationary nonequilibrium state with flowing current can be described by scattering from a single-particle potential that differs from the ground-state $\mathrm{KS}$ potential. Then the $V_{\mathrm{XC}}$ as appearing in Eq. (3) must correct the KS off-resonant transmission sufficiently to match that of the effective potential.

We conclude by noting that any formalism to treat a many-body problem that yields the Kubo response formula when analyzed within TDCDFT will recover Eq. (3). ${ }^{14,15}$

This work was supported by the DOE under Grant No. DE-FG02-01ER45928 and by the Deutsche Forschungsgemeinschaft. We thank Roberto Car, Achim Rosch, Peter Wölfle, and others for discussions.
${ }^{1}$ A. Nitzan and M. A. Ratner, Science 300, 1384 (2003).

${ }^{2}$ M. Brandbyge, J.-L. Mozos, P. Ordejon, J. Taylor, and K. Stokbro, Phys. Rev. B 65, 165401 (2002).

${ }^{3}$ P. S. Damle, A. W. Gosh, and S. Datta, Phys. Rev. B 64, 201403(R) (2001).

${ }^{4}$ S. T. Pantelides, M. Di Ventra, and N. D. Lang, Ann. N.Y. Acad. Sci. 960, 177 (2002).

${ }^{5}$ M. Di Ventra, S. T. Pantelides, and N. D. Lang, Phys. Rev. Lett. 84, 979 (2000).

${ }^{6}$ J. Heurich, J. C. Cuevas, W. Wenzel, and G. Schön, Phys. Rev. Lett. 88, 256803 (2002).

${ }^{7}$ Y. Q. Xue, S. Datta, and M. A. Ratner, Chem. Phys. 281, 151 (2002).

${ }^{8}$ F. Evers, F. Weigend, and M. Koentopp, Physica E (Amsterdam) 18, 255 (2003).

${ }^{9}$ R. Landauer, IBM J. Res. Dev. 1, 223 (1957).

${ }^{10}$ F. Evers, F. Weigend, and M. Koentopp, Phys. Rev. B 69, 235411 (2004).

${ }^{11}$ P. Hohenberg and W. Kohn, Phys. Rev. 136, B864 (1964).

${ }^{12}$ E. Runge and E. K. U. Gross, Phys. Rev. Lett. 52, 997 (1984).

${ }^{13}$ J. R. Reimers, Z.-L. Cai, A. Bilic, and N. S. Hush, Ann. N.Y. Acad. Sci. 1006, 235 (2003).
${ }^{14}$ P. Delaney and J. C. Greer, Phys. Rev. Lett. 93, 036805 (2004).

${ }^{15}$ K. Burke, R. Car, and R. Gebauer, Phys. Rev. Lett. 94, 159901(E) (2005).

${ }^{16}$ G. Stefanucci and C.-O. Almbladh, Europhys. Lett. 67, 14 (2004).

${ }^{17}$ G. Vignale and W. Kohn, Phys. Rev. Lett. 77, 2037 (1996).

${ }^{18}$ H. U. Baranger and A. D. Stone, Phys. Rev. B 40, 8169 (1989).

${ }^{19}$ A. Kamenev and W. Kohn, Phys. Rev. B 63, 155304 (2001).

${ }^{20}$ P. Bokes and R. W. Godby, Phys. Rev. B 69, 245420 (2004).

${ }^{21}$ J. P. Perdew, R. G. Parr, M. Levy, and J. L. Balduz, Jr., Phys. Rev. Lett. 49, 1691 (1982).

${ }^{22}$ S. Kummel, L. Kronik, and J. P. Perdew, Phys. Rev. Lett. 93, 213002 (2004).

${ }^{23}$ G. Vignale, C. A. Ullrich, and S. Conti, Phys. Rev. Lett. 79, 4878 (1997).

${ }^{24}$ N. Sai, M. Zwolak, G. Vignale, and M. Di Ventra, Phys. Rev. Lett. 94, 186810 (2005).

${ }^{25}$ J. Park et al., Nature (London) 417, 722 (2002); S. Kubatkin et al., Nature (London) 425, 698 (2003); H. Van der Zant, J. Am. Chem. Soc. (to be published).

${ }^{26}$ M. Koentopp, Dissertation Uni Karlsruhe, 2005. 